\title{
Features of point clouds synthesized from multi-view ALOS/PRISM data and comparisons with LiDAR data in forested areas
}

\author{
Wenjian $\mathrm{Ni}^{\mathrm{a}, \mathrm{b}, *}$, Kenneth Jon Ranson ${ }^{\mathrm{c}}$, Zhiyu Zhang ${ }^{\mathrm{a}}$, Guoqing Sun ${ }^{\mathrm{b}}$ \\ a State Key Laboratory of Remote Sensing Science, Institute of Remote Sensing and Digital Earth of Chinese Academy of Sciences, Beijing 100101, China \\ b Department of Geographical Sciences, University of Maryland, College Park, MD 20742, USA \\ c Code 618, Biospheric Sciences Branch, NASA/Goddard Space Flight Center, Greenbelt, MD 20771, USA
}

\section{A R T I C L E I N F O}

Article history:

Received 19 August 2013

Received in revised form 20 January 2014

Accepted 4 April 2014

Available online 3 May 2014

\section{Keywords:}

ALOS/PRISM

Point clouds

Synergy

Forest biomass

Forest vertical structures

\begin{abstract}
A B S T R A C T
LiDAR waveform data from airborne LiDAR scanners (ALS) e.g. the Land Vegetation and Ice Sensor (LVIS) have been successfully used for estimation of forest height and biomass at local scales and have become the preferred remote sensing dataset. However, regional and global applications are limited by the cost of the airborne LiDAR data acquisition and there are no available spaceborne LiDAR systems. Some researchers have demonstrated the potential for mapping forest height using aerial or spaceborne stereo imagery with very high spatial resolutions. For stereo images with global coverage but coarse resolution new analysis methods need to be used. Unlike most research based on digital surface models, this study concentrated on analyzing the features of point cloud data generated from stereo imagery. The synthesizing of point cloud data from multi-view stereo imagery increased the point density of the data. The point cloud data over forested areas were analyzed and compared to small footprint LiDAR data and large-footprint LiDAR waveform data. The results showed that the synthesized point cloud data from ALOS/PRISM triplets produce vertical distributions similar to LiDAR data and detected the vertical structure of sparse and non-closed forests at $30 \mathrm{~m}$ resolution. For dense forest canopies, the canopy could be captured but the ground surface could not be seen, so surface elevations from other sources would be needed to calculate the height of the canopy. A canopy height map with $30 \mathrm{~m}$ pixels was produced by subtracting national elevation dataset (NED) from the averaged elevation of synthesized point clouds, which exhibited spatial features of roads, forest edges and patches. The linear regression showed that the canopy height map had a good correlation with RH50 of LVIS data with a slope of 1.04 and $\mathrm{R}^{2}$ of 0.74 indicating that the canopy height derived from PRISM triplets can be used to estimate forest biomass at $30 \mathrm{~m}$ resolution.
\end{abstract}

(c) 2014 Elsevier Inc. All rights reserved.

\section{Introduction}

The accurate mapping of forest biomass over large areas is important for studies of global climate change and the carbon cycle. Recent progress in the estimation of forest biomass from remote sensing data is mainly due to the success in the extraction of related forest structure parameters. The data sources and methods for obtaining information on forest vertical structure can be summarized as the following four categories:

(1) LiDAR data is the best dataset for the estimation of forest biomass because it can directly measure the vertical structure of forests. Drake et al. (2002) explored the ability of large-footprint LiDAR instruments to estimate important structural attributes using the data acquired by the airborne Land Vegetation and Ice Sensor (LVIS). They reported that LVIS metrics were able to predict aboveground biomass with $\mathrm{R}^{2}=0.93$. Sun, Ranson, Kimes,

\footnotetext{
* Corresponding author at: State Key Laboratory of Remote Sensing Science, Institute of Remote Sensing and Digital Earth of Chinese Academy of Sciences, Beijing 100101, China.

E-mail address: niwj@radi.ac.cn (W. Ni).
}

Blair, and Kovacs (2008) found that LiDAR waveforms acquired by the spaceborne Geoscience Laser Altimeter System (GLAS) was highly correlated with that of LVIS with $\mathrm{R}^{2}=0.82$ for October 2003 GLAS data. Bortolot and Wynne (2005) developed an individual tree-based algorithm for determining forest biomass using small footprint LiDAR data. Pang, Lefsky, Miller, Sherrill, and Andersen (2008) explored the automatic tree crown delineation using discrete return LiDAR. Ni-Meister et al. (2010) assessed the application of vegetation structure parameter derived from LiDAR data for the mapping of aboveground biomass. Lefsky et al. (2005) estimated forest height and biomass using spaceborne Geoscience Laser Altimeter System (GLAS) data. Dubayah et al. (2010) estimated tropical forest height and biomass using LVIS. However, it is difficult to collect data at regional or global scales using airborne LiDAR because of its cost. GLAS acquired during the ICESat mission (2003-2009) had global coverage, but only provided point sampling data. Other imagery data is needed to extrapolate the samples to generate large area results.

(2) Forest structure can be extracted from the height of the scattering phase center (HSPC) of InSAR data. This requires knowledge 
of the elevation of the ground surface under the forest from other sources. Simard et al. (2006) mapped mean tree height in mangrove forests in the Everglades National Park using C-band HSPC of Shuttle Radar Topography Mission (SRTM) and Solberg et al. (2010) showed good results with X-band SRTM data. Kellndorfer et al. (2004) reported that the HSPC from SRTM with surface elevation from national elevation datasets (NED) could estimate vegetation height at Georgia and California sites with $\mathrm{R}^{2}=0.79$ and 0.75 , respectively. However, except for SRTM, most spaceborne InSAR data depend on repeat-pass operation and the quality of these data may be impacted by temporal decorrelation (Hall et al., 2011).

(3) The extraction of forest height through the combination of dual wavelength InSAR data. Neeff, Dutra, dos Santos, Freitas, and Araujo (2005) used the difference between digital surface models (DSM) from X-band and P-band InSAR data as a measure of vegetation height in the estimation of forest biomass. Balzter, Rowland, and Saich (2007) used X-band and L-band InSAR data acquired by E-SAR airborne sensors to estimate the top heights of forest stands. This research mainly utilized the dependence of penetration depth of SAR data on wavelength. These studies are only applicable at local scales due to the limited coverage of airborne data.

(4) Polarimetric Interferometric SAR (PolInSAR) is a promising technique for the estimation of forest height and biomass. PolInSAR employs the dependence of penetration depth of SAR on polarization (Cloude \& Papathanassiou, 2003; Garestier, DuboisFernandez, \& Papathanassiou, 2008; Papathanassiou \& Cloude, 2001). Temporal decorrelation is the main problem in the application of PolInSAR and limits the usefulness of the repeat pass InSAR data (e.g., Hall et al., 2011).

Besides the above four approaches, photogrammetry (or stereo imagery) is another technique which is directly sensitive to the forest vertical structure. Photogrammetry is a traditional technique for the extraction of a digital surface model (DSM). Information about forest canopy height should be contained in the stereo imagery because it is acquired by an optical sensor that records the signal mainly reflected by the top surface of the forest canopy. In the past, photogrammetry utilized aerial stereo images to map the elevation of the ground surface. The manual selection of matching ground points in the stereo pairs suppressed information about the forest structure. The high acquisition cost of aerial photos, the narrow swath, the complicated mathematical equations and the professional processing techniques of traditional photogrammetry based on film images hindered its application beyond surveying and mapping. Therefore, photogrammetry has not been used extensively for estimation of forest height and biomass.

The launch of spaceborne sensors, the application of digital cameras, the maturation of photogrammetry theory and the development of fully digital and automatic image processing make the application of photogrammetric methods easier. However, these advances occurred nearly in the same period with the appearance of LiDAR data. Many researchers working on forest height and biomass estimation began using LiDAR data. Nevertheless, some researchers in the field of surveying and mapping began to investigate the extraction of forest structure from stereo imagery at the beginning of this century. Sheng, Gong, and Biging (2001) successfully reconstructed the crown surface of a redwood tree from high-resolution aerial images. Gong, Mei, Biging, and Zhang (2002) presented a correction method for the improvement of the canopy boundary locations in the digital surface model (DSM) derived from high-resolution aerial images. Naesset (2002) measured the mean tree height of 73 forest stands in a 1000 ha forest area by automatic stereo processing of aerial images. Korpela (2004) explored the plausibility of the use of multi-scale aerial photographs to conduct forest inventory at the individual tree level. The results showed that very high accuracy at the individual tree level could not be expected while the aggregate stand variables could be estimated very accurately. Nuske and Nieschulze (2004) investigated the possibility to automatically derive a stand height for a dense beech stand using stereo imagery with a resolution of $0.44 \mathrm{~m}$. The canopy height model was derived by the difference between a DSM from stereo imagery and existing digital elevation model (DEM, i.e. the digital elevation model of ground surface under a forest canopy); St-Onge, Jumelet, Cobello, and Vega (2004) investigated the extraction of tree height through the combinations of the DEM from aerial stereo imagery and that from LiDAR data. StOnge, Vega, Fournier, and Hu (2008) created hybrid photo-LiDAR canopy height models (CHMs) by subtracting the LiDAR DEM from the aerial photogrammetric DSM. The results showed that the quality of photogrammetric DSM could be affected by sunlight and viewing geometry. Photo-LiDAR CHMs were well correlated to their LiDAR counterparts on a pixel-wise basis but have a lower resolution and accuracy. It also demonstrated that plot metrics extracted from the LiDAR and photoLiDAR CHMs, such as height at the 95th percentile of $20 \mathrm{~m} \times 20 \mathrm{~m}$ windows, were highly correlated. Toutin (2004) reported the results from IKONOS spaceborne stereo imagery with $0.8 \mathrm{~m}$ resolution. Hobi and Ginzler (2012) discussed the DSMs created from stereo imagery acquired by the spaceborne sensor WorldView- 2 with a resolution of $0.46 \mathrm{~m}$ at Nadir.

These studies demonstrated that the stereo imagery held great potential for deriving canopy height. However, most of the current studies were conducted on aerial images or spaceborne images with very high resolutions $(\leq 1.0 \mathrm{~m})$. The resolution of spaceborne stereo sensors with global coverage is not as high. The characteristics of DSMs could be directly affected by image resolution because the DSM from stereo imagery depends on the number and quality of the matching points from stereo images recognized automatically based on image textures. In addition, most current research in this area are mainly based on the digital surface model (DSM), which is an interpolation of the elevations of matching points.

The point cloud (PC) data (three-dimensional locations of matching points) similar to that of small footprint LiDAR data can be derived from stereo photogrammetry processing and has not been fully examined over forested areas. The methods used for analysis of LiDAR point cloud data can be applied to the point cloud of stereo imagery. However the acquisition of point clouds in photogrammetry is quite different from LiDAR so the indices derived from cloud data may have different information about forest structure.

Some spaceborne stereo sensors are composed of three independent optical systems for viewing nadir (N), forward (F) and backward (B) producing a set of stereo images along the satellite's track. In studies using multi-view stereo imagery, the DSMs derived from different combinations of stereo pairs were often simply averaged (Leberl et al., 2010). In fact, the different combinations of views have different information. For example, for a satellite in a descending polar-orbit, the forward sensor could see the north side of forest canopy (tree crowns); the backward sensor could see the south side of forest canopy while both sides are visible on nadir image. The point cloud from the image pair of forward and nadir (FN) mainly includes the points located on the north side of a forest canopy while that of nadir and backward (NB) should come from the opposite side. The point cloud from the image pair of forward and backward (FB) images should locate the top surface of a forest canopy. Therefore, the fusion of the three sets of point clouds could provide more information about the structure of forest canopy than any one of them because of the increased density of point cloud data.

The Panchromatic Remote-sensing Instrument for Stereo Mapping (PRISM) onboard the Advanced Land Observation Satellite (ALOS) has acquired multiple global coverages from June 2006 to April 2011. China launched their third Earth resources satellite Zi Yuan 03 (ZY03) on January 9, 2012.ZY03 is specifically designed for the collection of stereo imagery with a resolution of $3.6 \mathrm{~m}$ for forward and backward views and $2.1 \mathrm{~m}$ for the nadir view similar to ALOS/PRISM. Therefore, it is 
useful to explore the information contained in the point clouds of multiview stereo imagery for regional mapping of forest canopy height and biomass.

The test site and data used in this study will be described in Section 2. For the convenience of understanding, the basic principles of stereo imagery processing implemented in most software will be briefly reviewed in the first part of Section 3. The second part of Section 3 will describe the techniques used for analysis of point cloud data from multi-view stereo imagery. The features of point clouds over forested areas will be explored in Section 4 followed by discussion and conclusions.

\section{Test site and data}

\subsection{Test site}

The test site is located in a forested area near Howland, Maine USA $\left(45^{\circ} 08^{\prime} \mathrm{N}, 68^{\circ} 45^{\prime} \mathrm{W}\right)$. The stands in this northern hardwood boreal transitional forest consist of hemlock-spruce-fir, aspen-birch, and hemlock-hardwood mixtures. The common species include Populus tremuloides (quaking aspen), Betula papyrifera (paper birch), Tsuga canadensis (eastern hemlock), Picea rubens (red spruce), Abies balsamea (balsam fir), and Acer rubrum (red maple) (Huang et al., 2013). The region features relatively level and gently rolling topography where the elevation ranges from $40 \mathrm{~m}$ to $207 \mathrm{~m}$ within an area of $35 \mathrm{~km} \times 35 \mathrm{~km}$. This site has an Ameriflux tower situated within an intermediate aged forest (http://ameriflux.ornl.gov/fullsiteinfo.php?sid=54). The surrounding land is privately owned by a timber production company and has a history of multiple forest management manipulations, such as clear-cut, select-cut and strip-cut (Huang et al., 2013). This site is currently used for interdisciplinary forest research and experimental forestry practices with extensive field and airborne data acquired since 1989. This site is covered by both the LiDAR waveform data acquired by the NASA LVIS in 2003 and 2009 respectively and the high density point cloud data of small footprint LiDAR acquired by NASA Goddard's LiDAR, Hyperspectral \& Thermal Imager (G-LiHT) in June 2012. Therefore this site is very suitable to explore the features of points cloud from multi-view stereo imageries.

\subsection{Data}

\subsubsection{PRISM}

The ALOS/PRISM data is used in this study due to its global coverage and potential for regional mapping of forest canopy height and biomass. PRISM is a panchromatic radiometer with a $2.5 \mathrm{~m}$ spatial resolution at nadir. PRISM has three independent optical systems for viewing in the nadir, forward and backward directions along the satellite orbit track. Forward and backward telescopes are inclined +24 and $-24^{\circ}$ from nadir to realize a base-to-height ratio of 1.0. The nadir-viewing telescope covers a swath width of $70 \mathrm{~km}$; whereas forward and backward telescopes cover a swath of $35 \mathrm{~km}$. The wide field of view (FOV) provides three fully overlapped stereo (triplet) images of a $35 \mathrm{~km}$ width without mechanical scanning or yaw steering of the satellite. PRISM has 9 observation modes resulting from different combinations of the three views. The data used in this study were acquired on 09/05/2009 under the triplet observation mode using all the three views (i.e., PRISM Mode 1) with a swath width of $35 \mathrm{~km}$. It was processed to level 1B1 which is radiometrically calibrated at the sensor. WGS84/EGM96 was used for horizontal and elevation reference of DSMs and point clouds, as well as the ground control points used in the calculation of rational polynomial coefficients (RPC).

\subsubsection{G-LiHT}

G-LiHT is a portable airborne system developed by the NASA Goddard Space Flight Center that simultaneously maps the composition, structure and function of terrestrial ecosystems (Cook et al., 2013). GLiHT is composed of a scanning LiDAR, imaging spectrometer and thermal camera to fulfill the data coincidence in time and space for data fusion. The G-LiHT airborne laser scanner (VQ-480, Riegl Laser Measurement Systems, Horn, Austria) uses a $1550 \mathrm{~nm}$ laser that provides an effective measurement rate of up to $150 \mathrm{kHz}$ along a 60degree swath perpendicular to the flight direction. At a nominal flying altitude of $335 \mathrm{~m}$, each laser pulse has a footprint of $\sim 10 \mathrm{~cm}$ diameter and is capable of producing up to 8 returns. Classified point cloud data and digital terrain and canopy height models are openly distributed on the G-LiHT webpage (http://gliht.gsfc.nasa.gov). The elevations are in meters referenced to the WGS84/EGM96 geoid.

\subsubsection{NED}

The national elevation dataset (NED) is the primary elevation data product of the Unite States Geologic Survey (USGS). This product is distributed in geographic coordinates in units of decimal degrees. Its horizontal reference is the North American Datum of 1983 (NAD 83) and its vertical reference is the North American Vertical Datum of 1988 (NAVD 88). The vertical reference of NED data were transformed to the WGS84/ EGM96 using GEOID12 software developed by the National Geodetic Survey (NGS) and the GEOTRANS software.

\subsubsection{LVIS}

NASA's LVIS is an airborne laser altimeter system designed, developed and operated by the Laser Remote Sensing Laboratory at Goddard Space Flight Center. LVIS obtained sub-canopy and canopy-top topography data as well as canopy vertical structure information for forested sites at our study area in order to generate detailed forest structural data sets. The LVIS data used in this study were acquired in August, 2009 using a nominal footprint size of $20 \mathrm{~m}$. The LVIS data were released as three products, i.e. LVIS ground elevation (LGE), LVIS geolocated waveform (LGW) and LVIS canopy top elevation (LCE). The LGE includes location (latitude/longitude), ground surface elevation, and the heights of the energy quartiles (relative height to ground surface; RH25, RH50, RH75, and RH100) where $25 \%, 50 \%, 75 \%$ and $100 \%$ of the waveform energy occur. The LGW provides the geocoded waveforms of each LiDAR shot. The RH50 of LVIS LGE data was rasterized to $30 \mathrm{~m} \times 30 \mathrm{~m}$ pixels for evaluating canopy height from PRISM and NED in following sections. The RH50 observations for footprints located within a pixel were averaged to be the value of the pixel. The pixels having no footprints were masked out in the analysis. The elevation reference of LVIS data is transformed from WGS-84 (i.e., GRS80) ellipsoid to the WGS84/EGM96 geoid using the GEOTRANS software developed by the National GeospatialIntelligence Agency.

\section{Method}

\subsection{The processing of stereo imagery}

\subsubsection{Sensor model}

One critical component of photogrammetry is the sensor model which establishes the functional relationship between the twodimensional image space (line, sample) and the three-dimensional object space (latitude, longitude, elevation) by defining the location and viewing direction (i.e. the imaging geometry including pitch, roll and yaw angles) of the camera in space (Tao \& Hu, 2001). There are typically two categories of sensor models, i.e. physical and generalized models. Physical models are rigorous and normally yield high modeling accuracy. However, a physical sensor model represents the physical imaging process and contains some critical sensor technical information which is held as confidential by some data providers, especially commercial satellite data vendors (Tao \& Hu, 2001). In a generalized sensor model, the transformations between the image and the object space are represented as general functions, such as polynomials or rational functions, without modeling the physical imaging process. The rational function 
model (RFM) is a generalized sensor model, which uses ratios of polynomials to establish the relationship between the two-dimensional coordinates of the image of an object and the coordinates of the object in three-dimensional space. The physical parameters related to critical sensor information cannot be easily recovered from the RFM. In addition, the generalized sensor model is not sensor-dependent so that general processing software can be used to process the stereo imagery from different platforms. Only the rational polynomial coefficients (RPC) of the RFM need to be updated. Therefore the RFM is attractive and used in the state-of-art digital photogrammetric software packages.

In the rational function model, image pixel coordinates $(r, c)$ are expressed as the ratios of polynomials of ground coordinates $(X, Y, Z)$. In order to minimize the errors during the computations and improve the numerical stability of the equations, the two image coordinates and three ground coordinates are normalized to the range of -1.0 to +1.0 (Tao \& Hu, 2001). The RFM can be expressed as (Tao \& Hu, 2001):

$$
\begin{gathered}
r_{n}=\frac{r-r_{0}}{r_{s}}, c_{n}=\frac{c-c_{0}}{c_{s}}, X_{n}=\frac{X-X_{0}}{X_{s}}, Y_{n}=\frac{Y-Y_{0}}{Y_{s}}, Z_{n}=\frac{Z-Z_{0}}{Z_{s}} \\
r_{n}=\frac{p_{1}\left(X_{n}, Y_{n}, Z_{n}\right)}{p_{2}\left(X_{n}, Y_{n}, Z_{n}\right)} \quad c_{n}=\frac{p_{3}\left(X_{n}, Y_{n}, Z_{n}\right)}{p_{4}\left(X_{n}, Y_{n}, Z_{n}\right)},
\end{gathered}
$$

$$
\begin{aligned}
p_{l} & =a_{1, l}+a_{2, l} X_{n}+a_{3, l} Y_{n}+a_{4, l} Z_{n}+a_{5, l} X_{n} Y_{n}+a_{6, l} X_{n} Z_{n}+a_{7, l} Y_{n} Z_{n} \\
& +a_{8, l} X_{n}^{2}+a_{9, l} X_{n}^{2}+a_{10, l} Z_{n}^{2}+a_{11, l} X_{n} Y_{n} Z_{n}+a_{12, l} X_{n}^{3}+a_{13, l} X_{n} Y_{n}^{2} \\
& +a_{14, l} X_{n} Z_{n}^{2}+a_{15, l} X_{n}^{2} Y_{n}+a_{16, l} Y_{n}^{3}+a_{17, l} Y_{n} Z_{n}^{2}+a_{18, l} X_{n}^{2} Z_{n}+a_{19, l} Y_{n}^{2} Z_{n} \\
& +a_{20, l} Z_{n}^{3}
\end{aligned}
$$

where $l=1,2,3,4 . r_{0}, c_{0}$ and $r_{s}, c_{s}$ are the offsets and scale values for the normalization of image coordinate respectively while $X_{0}, Y_{0}, Z_{0}$ and $X_{s}$, $Y_{S}, Z_{s}$ are respectively the offsets and scale values for the normalization of ground coordinates. $P_{l}$ defined the transformation from $X_{n}, Y_{n}, Z_{n}$ to $r_{n}, c_{n}$ by the 20-term cubic form polynomial above. Therefore the RPC is always composed of these 90 coefficients. The RPC is provided in some stereo images and could be calculated using ground control points (GCP) by direct or iterative least squares methods as given in (Tao \& Hu, 2001). The two images in a stereo pair are referred to as left and right images respectively in traditional photogrammetry. Both images have their own RPCs.

\subsubsection{Stereo processing}

With the sensor model, a point in object space can easily be transformed into image space. However, the transformation from image space to object space needs the information of elevation. The central idea of photogrammetry is to determine the elevation of an object (or an image pixel) by measuring the displacement or difference in the apparent position of an object viewed along two different lines of sight (i.e., the parallax) of the matching points of the object on two stereo images. The two matching points for an object should be identified first to measure their parallax. The identification of matching points is a process of two-dimensional image matching based on the spatial characteristics of the two stereo images. An important advance in digital photogrammetry is the introduction of epipolar geometry (or epipolar constraints). By the epipolar geometry for a ground point on one image, the position of its projection (or matching point) on the other image is not random within the two-dimensional image space but on a line referred to as the epipolar line in the image space. The ground point and its projection centers of two images compose a plane in three-dimensional object space referred to as epipolar plane. The intersection between the images and epipolar plane are the epipolar lines in the images. For a given point on one image we only need to search its matching point along its epipolar line on the other image. Therefore the epipolar geometry greatly improves the computation efficiency by reducing the searching process from two-dimensional to one-dimensional space.
The epipolar geometry further indicates that the epipolar lines will be parallel with each other if the inclined imaging plane is transformed into the horizontal plane using the imaging geometry expressed by the senor models. The transformation of inclined image plane to horizontal image plane can be expressed as

$$
\left[\begin{array}{l}
x_{i}^{\prime} \\
y_{i}^{\prime} \\
z_{i}
\end{array}\right]=\left[\begin{array}{ccc}
\cos \kappa_{i} & -\sin \kappa_{i} & 0 \\
\sin \kappa_{i} & \cos \kappa_{i} & 0 \\
0 & 0 & 1
\end{array}\right]\left[\begin{array}{ccc}
1 & 0 & 0 \\
0 & \cos \omega_{i} & -\sin \omega_{i} \\
0 & \sin \omega_{i} & \cos \omega_{i}
\end{array}\right]\left[\begin{array}{ccc}
\cos \varphi_{i} & 0 & -\sin \varphi_{i} \\
0 & 1 & 0 \\
\sin \varphi_{i} & 0 & \cos \varphi_{i}
\end{array}\right]\left[\begin{array}{l}
x_{i} \\
y_{i} \\
f
\end{array}\right]
$$

where $\varphi, \omega$ and $\kappa$ are the roll, pitch and yaw angles which can be calculated from the sensor model. $i=1$ or 2 represents the left and right images respectively. $f$ is the focal length of the camera. $\left[x_{i}, y_{i}\right]^{T}$ is the coordinate of a pixel in inclined image while $\left[x_{i}^{\prime}, y_{i}^{\prime}\right]^{T}$ is the corresponding coordinate of the pixel in horizontal image. $z_{i}^{\prime}$ should be zero or a constant in the horizontal image. After both inclined images are transformed to their corresponding horizontal images using Eq. (2), the right horizontal image will be further transformed to the left horizontal images by a rotation and translation:

$$
\left[\begin{array}{l}
x_{2}^{\prime \prime} \\
y_{2}^{\prime}
\end{array}\right]=\left[\begin{array}{cc}
\cos \theta & -\sin \theta \\
\sin \theta & \cos \theta
\end{array}\right]\left[\begin{array}{l}
x_{2}^{\prime} \\
y_{2}^{\prime}
\end{array}\right]+\left[\begin{array}{l}
x_{0} \\
y_{0}
\end{array}\right]
$$

where $\theta$ and $\left[x_{0}, y_{0}\right]^{T}$ are rotation angle and translation vector of the right horizontal image relative to the left. They need to be determined by some tie points identified from the two original stereo images by two-dimensional image matching or by manual selection. By applying Eqs. (2) and (3) with the tie points and sensor model, the two images of the stereo pair can be transformed into epipolar space, and are referred to as epipolar images. The identification of matching points can be automatically conducted line by line between the epipolar images. The elevation of ground points can be calculated by the parallax of the identified matching points using simple geometric relations (Jan, 1993). The point clouds are the collection of all the pixels with identified matching points. The digital surface model will be produced through the interpolation of the point clouds.

In the above processing, the three-dimensional positions of pixels were calculated based on the left image while the right image only provides the parallax information. Therefore the geometrical features of point clouds are the same as the left image. In this study the output of processing a stereo image pair includes three products: left image, point clouds and digital surface model.

\subsection{The synthesis of multi-stereo point clouds}

As mentioned above, ALOS/PRISM has three independent optical systems for viewing nadir (N), forward (F) and backward (B). Three different combinations of these images (FN, FB and NB) can be used to generate three different point cloud datasets. The synergy of these point clouds increases the density of the points to provide adequate information on the vertical structure of the ground objects.

\subsubsection{Co-registration of point clouds}

One basic prerequisite for the development of point clouds from different view combinations is that they must be accurately coregistered. Although the RFM is a good theoretical model of the imaging geometry of a sensor, the RPC is not error-free because it is determined by the measurement of sensor imaging parameters or ground control points. The uncertainties in the imaging parameter measurements or the coordinates of ground control points will inevitably be propagated to the RPC and finally be exhibited in the geocoding accuracy of stereo images, especially for high resolution images. An accurate co-registration method has been developed in our previous studies (Ni, Sun, Zhang, Guo, \& He, 2014a). The 
image to be resampled is referred to as the slave image, whereas the other image is referred to as the master image. It models the misregistration between master and slave images caused by translation, scale, skew, and rotation as

$\left[\begin{array}{l}x_{m} \\ y_{m}\end{array}\right]=A\left[\begin{array}{l}x_{s} \\ y_{s}\end{array}\right]+\left[\begin{array}{l}x_{0} \\ y_{0}\end{array}\right]$ where $A=\left[\begin{array}{cc}\cos \varphi & \sin \varphi \\ -\sin \varphi & \cos \varphi\end{array}\right]\left[\begin{array}{cc}k_{x} & w \\ 0 & k_{y}\end{array}\right]$

where $\left(x_{0}, y_{0}\right)^{\mathrm{T}}$ is the translation vector, $\left(k_{x}, k_{y}\right)$ are the scale factors along the sample and line directions, $w$ is the skew term, and $\varphi$ is the rotation angle. $\left(x_{m}, y_{m}\right)^{\mathrm{T}}$ are the center coordinates of a chip in the master image, whereas $\left(x_{s}, y_{s}\right)^{\mathrm{T}}$ are the center coordinates of a chip in the slave image having maximum correlation with the master chip. The control points of these two images were searched automatically based on the correlation of image chips, and the point qualities were screened based on the signal-to-noise ratio. To achieve successful coregistration, the number of control points should be much more than the number of parameters to be determined. The over-determined system of linear equations can be solved using the least squares method. The left image was used as master image in the co-registration process. The point clouds and DSMs are then resampled using the same coregistration parameters for their left images.

\subsubsection{Removal of elevation divergence}

Besides the spatial displacement, the divergence between the elevations from different view combinations could also be expected because of the errors associated with the RPCs. The accuracy of ground control points also affects the estimation of imaging geometry. The elevation variation caused by errors of these three angles would not be local ridges or valleys but overall surface inclination or distortion. In other words, the influence of errors of the three angles on elevation measurements should have low spatial frequency. Therefore, low-pass filtering could reveal the divergence between the elevations from different view combinations. A method has been proposed in our previous studies for the removal of atmospheric affect on the phase image of interferometric synthetic aperture radar which also showed the feature of low spatial frequency $(\mathrm{Ni}$, Sun, Zhang, Guo, \& He, 2014b). An essential step in low-pass filtering is the determination of the window size. If the window size is too small, most high spatial frequency details will remain in the filtered results. As the window size increases, the high frequency details in the filtered image will decrease. When the window size is large enough, the filtered image contains only the low frequency features. The changes in the standard deviation of the filtered image will become more stable as the window size increases. The window size of the filter was selected based on the where the standard deviation stabilized. The low-frequency features were then removed from these PC data and their DSMs.

\subsubsection{Rectification to other source DEM}

The results from different view combinations should have been accurately co-registered with each other after the two-steps described above. Both FB and NB have been co-registered to FN and the elevation divergences have been removed. Therefore the three sets of point clouds could be merged to produce the synthesized point cloud. The elevation data from other-sources, such as National Elevation Dataset

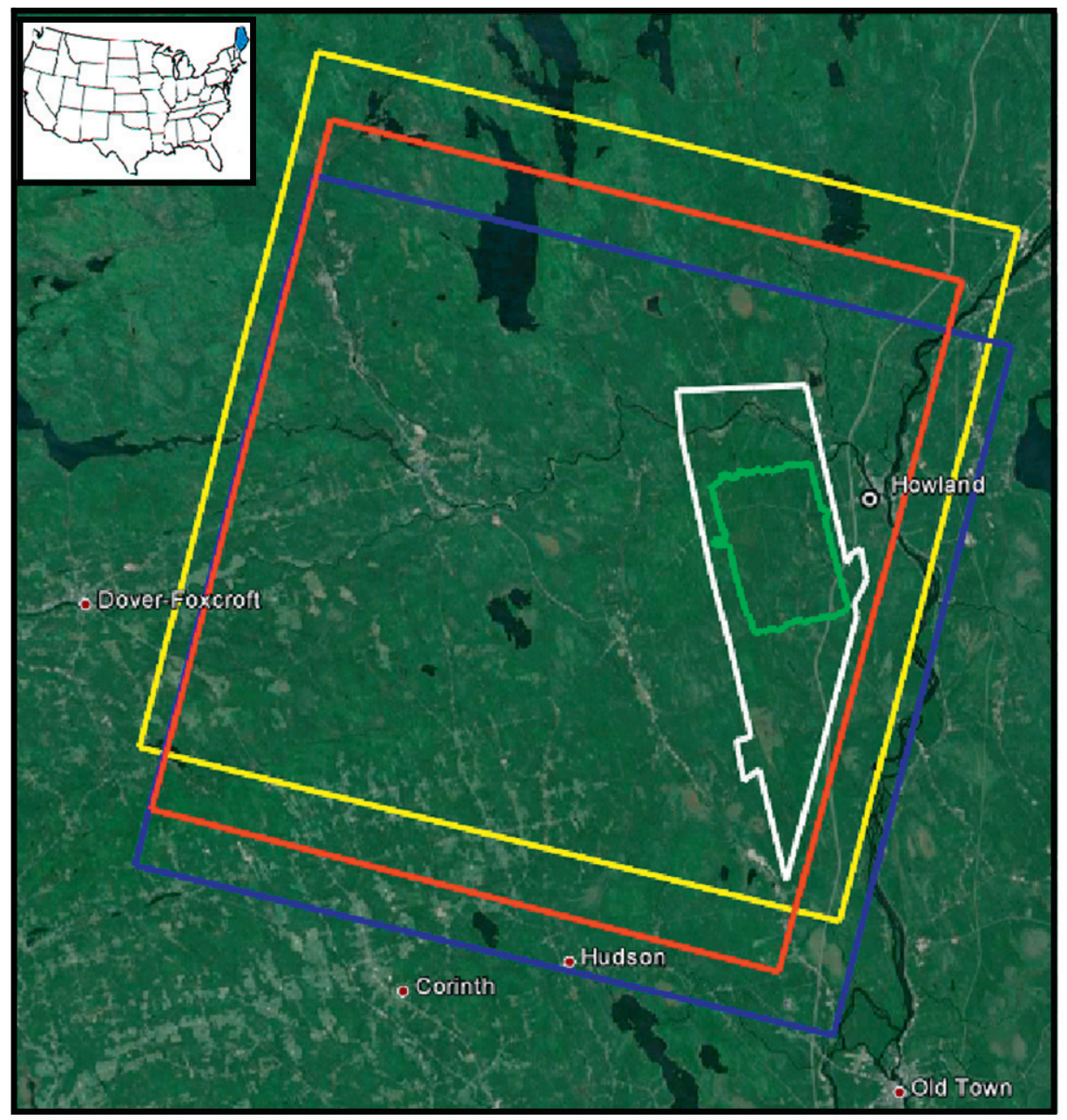

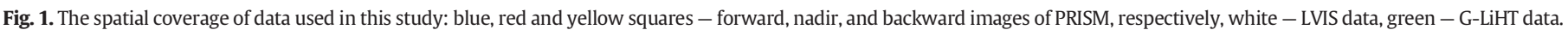
The background is true color image from Google Earth. 
(NED), have been used as the ground surface height to extract vegetation height from the elevation of stereo imagery (Ni, Sun, \& Ranson, 2013). As described in the previous two sections, the geocoding accuracy and elevation of stereo imagery data are inevitably affected by the accuracy of RPC. Therefore, the synthesized point cloud should be firstly co-registered to NED using Eq. (4) as in our previous studies (Ni et al., in press-a). Then the elevation divergence can be removed by lowspatial filtering as described in the above section. By these two steps the synthesized point clouds should be accurately co-registered and rectified to the other-source DEM and the heights of these points above the DEM can be generated.

\subsection{Analysis scheme}

Fig. 1 shows the spatial coverage of the related dataset within the test site. The features of point clouds from ALOS/PRISM were explored by comparisons with LVIS waveforms and G-LiHT point clouds over the footprint of LVIS $(20 \mathrm{~m})$. The resolution of products from the stereo processing of PRISM data is $3 \mathrm{~m}$. The pixels located within a $7 \times 7$ (i.e. $21 \mathrm{~m} \times 21 \mathrm{~m}$ ) window centered in an LVIS footprint were used to make the comparisons. In the best cases (all pixels have matching points in the stereo imagery), there will be 147 points within this $7 \times 7$ window ( 49 points in each view). In the vertical distribution histogram of the PRISM point cloud, the bin was set to $1 \mathrm{~m}$. The bin size for the vertical distribution histogram of the G-LiHT point cloud was set to $30 \mathrm{~cm}$ which is the same as the bin size of LVIS (Sun et al., 2008).

Averaging the heights of all points within a resolution grid of $30 \mathrm{~m}$ produced an elevation map from ALOS/PRISM. A canopy height map was further generated from the elevation map of ALOS/PRISM by taking the NED as the ground surface elevation as in many other studies (Kellndorfer et al., 2004). The comparisons between the canopy height map and RH50 of LVIS data at a resolution of $30 \mathrm{~m}$ were also made because RH50 is a good indicator of forest biomass (Huang et al., 2013).

\section{Results}

\subsection{Removal of elevation divergences}

As described in the previous section, the RFM model (i.e. RPC) of each view was reconstructed using ground control points. The same set of ground control points was used for all three views to minimize the influence of ground control points on the data synthesis. There were 1768 points selected by the automatic image matching with accuracy better than 0.1 pixels. Table 1 lists the estimated imaging geometry of the three views and their corresponding accuracy. The accuracy was depicted by the root mean square errors of predicted image coordinates.

Fig. 2(a)-(c) shows the elevation difference between FB and FN (a), NB and FN (b) as well as between FN and NED (c) after they were accurately co-registered with each other. The high-frequency spatial changes in (a)-(c) are caused by local objects such as trees seen from different directions, while the low-frequency changes in (a)-(c) by errors of imaging geometry used in stereo image processing which need to be removed. The low-pass filtering was applied to the resized elevation difference images with a factor of 0.1 to reduce the computation load. Since the original pixel size of PRISM elevation images is $3 \mathrm{~m}$, the

Table 1

The estimated imaging geometry of different views using ground control points.

\begin{tabular}{lrrllll}
\hline \multirow{2}{*}{ View } & \multicolumn{2}{l}{ Imaging geometry $\left({ }^{\circ}\right.$, degree) } & & \multicolumn{2}{l}{ Accuracy (in pixel) } \\
\cline { 2 - 3 } & Roll $(\varphi)$ & Pitch $(\omega)$ & Yaw $(\kappa)$ & & RMSX & \multicolumn{2}{c}{ RMSY } \\
\hline Forward & -0.114778 & -23.996583 & -12.776417 & & 0.02743 & 0.00748 \\
Nadir & 0.167656 & 0.092828 & -14.512201 & & 0.00470 & 0.00811 \\
Backward & 0.254939 & 24.228831 & -13.142084 & & 0.03632 & 0.00794 \\
\hline
\end{tabular}

pixel size of images shown in Fig. 2 is about $30 \mathrm{~m}$. Fig. 2(d)-(f) shows the changes of standard deviation with respect to the window size used in lowpass filtering, corresponding to Fig. 2(a)-(c) respectively. The window size of $71 \times 71$ was used for (a) and (b) and $181 \times 181$ for (c) because the standard deviation become stable after these window sizes ( $\mathrm{Ni}$ et al., 2014b). Fig. 2(g)-(i) were the results of Fig. 2(a)-(c) after the removal of elevation divergences using their corresponding window size. It was clear that the elevation divergences caused by the error of the RFM model was successfully removed.

\subsection{Analysis of point clouds}

Fig. 3(a) presents the horizontal distributions of point clouds from different view combinations as different colors. The Red, Green and Blue colors were assigned to pixels matched in stereo processing of $\mathrm{NB}, \mathrm{FN}$ and FB combinations, respectively. If a pixel had no matching pixel its value was set to zero, so the dark pixel in Fig. 3 indicates that it could not be recognized from all stereo combinations while the white pixel indicated that it could be recognized in all of the three view combinations. The color of a pixel depends on which of the three view combinations was valid for calculating the height of the pixel. The colored image shows the spatial complementation of point clouds from multi-views. Fig. 3(b) and (c) are the enlargements over a lake area and clear-cut patches, respectively. The white lines that appear on the boundary of water bodies and shore, around clear cut patches, and roads indicate that object edges were well defined in the stereo imagery.

The vertical lines evenly spaced across the epipolar images shown in this figure might be a specific effect of algorithms used in the searching of matching points by the software because no such feature existed in the stereo image pair. No visible effect was observed on the difference image of two DSMs as well as the final canopy height map from the stereo data.

Fig. 4 compares the point clouds from ALOS/PRISM with the point cloud data from G-LiHT and LVIS waveforms over typical forest stands. Each row represents a forest stand. The middle column (e, f, g and h) is the canopy height model $(\mathrm{CHM})$ from G-LiHT $(21 \times 21$ grids $)$ with a grid size of $1 \mathrm{~m}$ centered at a LVIS footprint. The spatial patterns of canopy height shown in Fig. 4(e), (f), (g) and (h) indicate that these four cases represent 1) two-layer dense forest (at elevations of $\sim 90 \mathrm{~m}$ and $100 \mathrm{~m}$ ), 2) disturbed forest (tall canopy at elevation of $\sim 124 \mathrm{~m}$ with gaps at $\sim 108 \mathrm{~m}$ ), 3) sparse forest (several small trees with heights 6-9 m) and 4) uniform dense forest (no gaps, the ground elevation at this spot is $\sim 90 \mathrm{~m}$, so the tree height ranges from 10 to $20 \mathrm{~m}$ ), respectively.

The left column ( $a, b, c$ and d) compares the vertical histograms of point clouds from PRISM (red - NB, yellow - FN, blue - FB), vertical height profile (histogram) of G-LiHT PC data (green curve) in the $21 \mathrm{~m} \times 21 \mathrm{~m}$ grid, LVIS waveform (pink curve), and shows the elevation of NED, mean PRISM, and LVIS height metrics (ground, RH50 and RH100) by horizontal lines of solid black, dotted black, solid red, thin solid green and thin dotted green, respectively. The horizontal axis of the plots in this column is the DN of the LVIS waveform. The point number in each bin of ALOS/PRISM and G-LiHT data were scaled using their corresponding ratios shown in the plots to make the peaks of PRISM, G-LiHT histograms the same as the LVIS data. The number of points from NB, FB and FN was plotted as red, yellow and blue respectively to show the contributions from different view combinations. The text at the top-center of the plots shows the ID and shot number of the LVIS laser pulse, latitude/longitude of the LVIS footprint, and the line/sample of the LVIS center on the PRISM image. The text at the bottom of each plot shows ratios used to normalize the histograms of PRISM and G-LiHT, numbers of points of PRISM PC data in the $21 \mathrm{~m}$ by $21 \mathrm{~m}$ window, the elevations of ground surface, RH50 and RH100 of the LVIS waveform, and the NED elevation, mean height of PRISM PC. Fig. 4 shows that the point clouds of ALOS/PRISM had different features over these four forest conditions. The first layer could be captured by the 

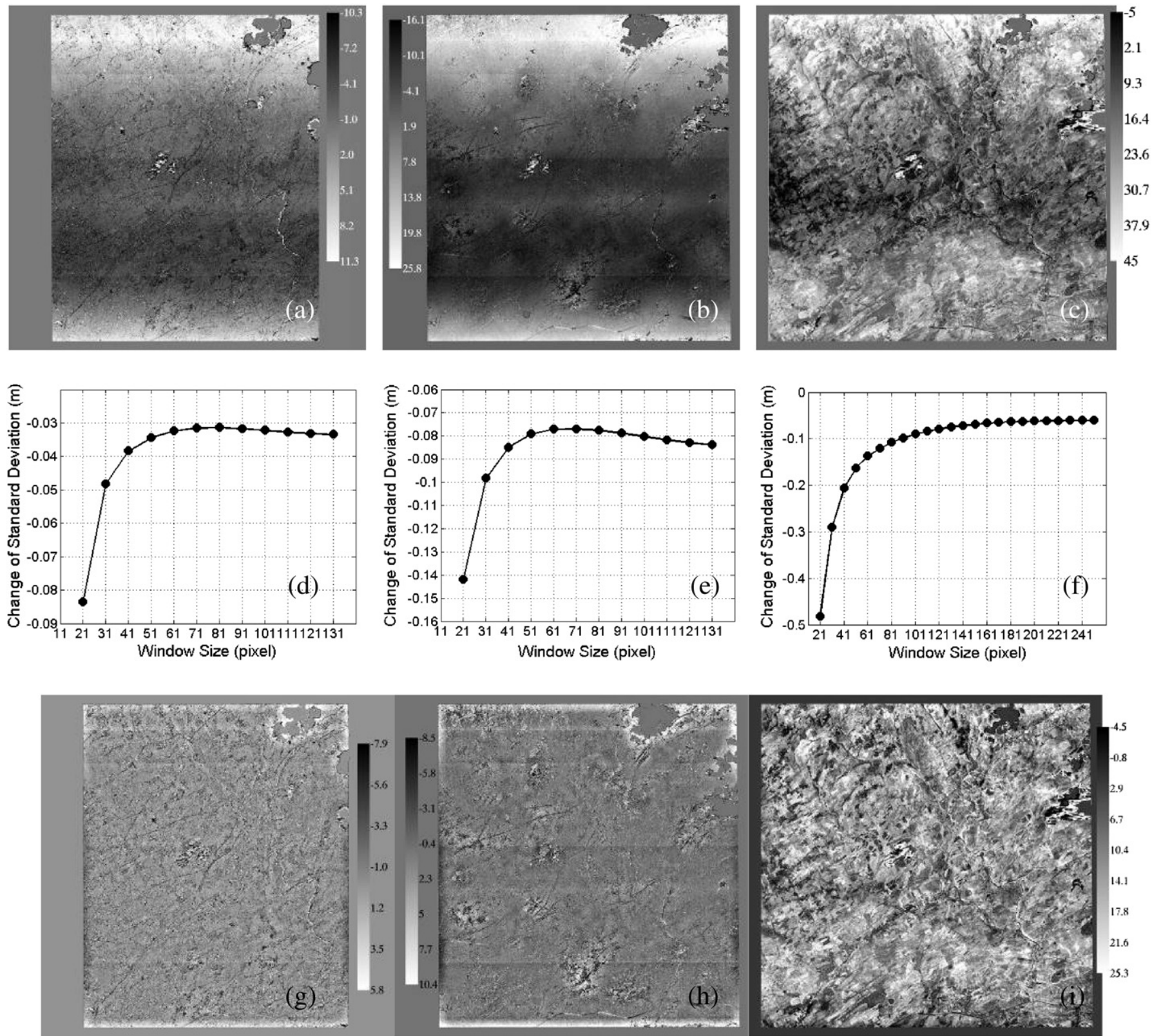

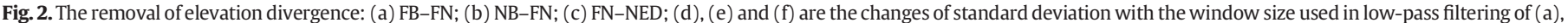
(b) and (c) respectively. (g), (h) and (i) were the results of (a), (b) and (c) after the removal of elevation divergence respectively.

point clouds of ALOS/PRISM as shown in Fig. 4(a) while the second layer could not be seen. The point clouds of ALOS/PRISM scattered from the forest canopy to the ground surface when the forest canopy was not closed as shown in Fig. 4(b). One common characteristics observed in Fig. 4(a), (b) and (c) was that point clouds of ALOS/PRISM could not capture the maximum height of forest stands as shown by G-LiHT. This feature could be an interpretation for the underestimation of forest height as reported in other studies. The maximum height (top of trees) could be missed because of the relatively large pixel size (2-3 m). Some outlier points above the maximum height could be observed in uniform forest areas as shown in Fig. 4(d). This phenomenon was expectable because the identification of matching points was based on the automatic image matching along epipolar lines, which tends to fail in cases of low image texture. There were only 24 points recognized in stereo matching which was about $1 / 3$ of those in the other three forest cases. This phenomenon also occurred on bare flat ground, which also had low texture. Another important phenomenon in all of these four cases was that the vertical distribution of point clouds from different view combinations did not coincide. The higher point was not seen from the combination of FB, but it is seen in combinations of NB and FN. This demonstrates the complementarity of point clouds from different view combinations. For all four forest cases shown here the mean height of PRISM point cloud data is greater than RH50 and less than RH100 of LVIS data. Since the RH100 represents the top canopy height and RH50 has been used successfully for forest biomass estimation, the comparisons of PRISM height with RH50 and RH100 will reveal the feature and usefulness of the height information derived from PRISM PC data (see Fig. 5 below).

The right column ( $\mathrm{i}, \mathrm{j}, \mathrm{k}$ and $\mathrm{l}$ in Fig. 4) compares the vertical profiles of point clouds with G-LiHT profiles over $100 \mathrm{~m}$ rectangles using the same center as Fig. 4(a), (b), (c) and (d), respectively. The horizontal axis of the third column is the number of points from ALOS/PRISM. The number of points from G-LiHT was normalized to the same range using a ratio of the peaks of the two histograms. As shown in these plots, the vertical distributions of point clouds over large spatial sales ( $100 \mathrm{~m}$ ) were similar to that at the scale of an LVIS footprint. The vertical 

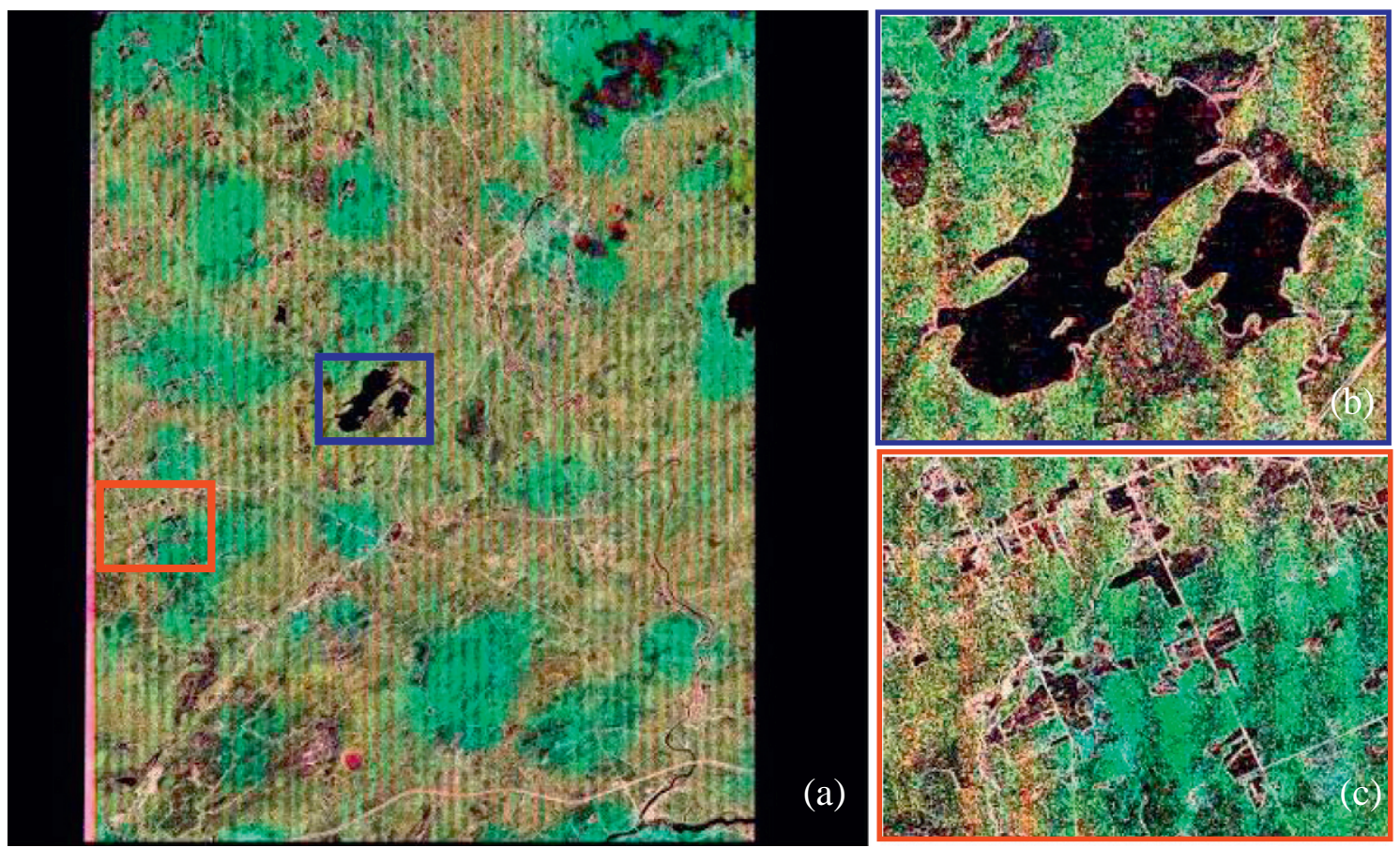

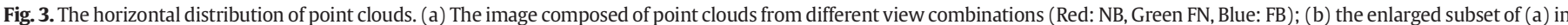
the blue rectangle; (c) the enlarged subset of (a) in the red rectangle.

distributions of point clouds from different view combinations over large spatial scales were similar for dense forest as shown in Fig. 4(i) and (1). However, it is quite different for more open forest canopies as shown in Fig. 4(j) and (k). For forested areas (Fig. 4(i), (j) and (1)) the ascending edge of vertical profiles of point clouds from ALOS/PRISM resembles that of G-LiHT very well. This feature indicates that more information could be extracted from PRISM point cloud by constructing useful height indices as those from LiDAR point clouds. For dense canopies as shown in Fig. 4(i) and (1), the ground surface cannot be identified from PRISM PC data.

\subsection{Canopy height map}

Fig. 5(a) shows a subset of canopy height map at a resolution of $9 \mathrm{~m}$ derived by subtracting NED from the average height of point clouds of multi-view combinations. Fig. 5(b) is the panchromatic image from nadir view PRISM data covering the same area as Fig. 5(a). The spatial features of roads, forest edges, patches and strip cuts at different directions shown in Fig. 5(b) are also clearly exhibited in Fig. 5(a). Fig. 5(c) shows the forest canopy height map of the entire PRISM scene at a resolution of $30 \mathrm{~m}$. The blue rectangle in Fig. 5(c) is the enlarged area in Fig. 5(a) and (b). The yellow polygon is the area covered by LVIS data. Fig. 5(d) is the enlargement of the yellow polygon area in Fig. 5(c). Fig. 5(e) is RH50 of LVIS data. The spatial pattern of Fig. 5(d) resembled that of Fig. 5(e) very well. Fig. 5(f) is the scatter plot of PRISM canopy height vs. LVIS RH50 shown in Fig. 5(d) and (e). The slope of the linear regression was 1.04 with $R^{2}$ of 0.74 . Fig. $5(\mathrm{~g})$ is the scatter plot of PRISM canopy height vs. LVIS RH100. The slope of the linear regression was 0.67 with a $\mathrm{R}^{2}$ of 0.57 . Apparently, canopy height index (mean height of the point cloud) derived from the PRISM triplet and NED is higher than RH50 while lower than RH100 and has better correlation with RH50 than RH100. These results clearly demonstrated that canopy height index derived from PRISM triplet and NED had comparable information for forest biomass as RH50 of the LVIS data.

\section{Discussion}

In this study, the synthesis of point clouds from combinations of multi-view stereo imagery was conducted in epipolar space, as shown in Figs. 2 and 3, not in object space as those shown in Fig. 5(a) and (c) (geocoded). The processing in epipolar space enables the easy visualization and examination of the features of epipolar lines. Besides, the errors introduced by geocoding were excluded and the storage requirement was reduced by minimizing void pixels.

Two steps of processing, i.e. the accurate co-registration and removal of elevation divergence, were used for the synthesis of point clouds from multiple combinations of multi-view stereo imagery. As noted in the previous section, the mis-registration and elevation divergence were caused by the error of RPC. Accurate co-registration was always necessary especially for high-resolution image. The removal of elevation divergence might not be necessary if the accuracy of RPC was high enough, which could be judged by whether systemic offsets, inclinations or periodic black/white patterns could be observed in the difference image of two DSMs.

Fig. 4(b), (c), (j) and (k) showed that some points near or on the ground surface might be identified from the point clouds of ALOS/ PRISM. This feature indicates that it might be possible to derive heights of sparse or unclosed forests solely based on point clouds of ALOS/ PRISM. However, it is difficult to derive the maximum tree height because the canopy peak is always missed when the image resolution is not high enough.

The mean height of PRISM PC in a grid (e.g. $21 \mathrm{~m}$ shown in Fig. 4) is not the top canopy height $(<\mathrm{RH} 100)$ and higher than the RH50. At a large grid, such as $100 \mathrm{~m}$, the resemblance of the vertical distribution of ALOS/PRISM over dense forest with those of G-LiHT point clouds indicates that the vertical distribution of PRISM PC may be used to derive more height indices for characterization of forest spatial structure and carbon storage, which will be pursued in our future studies.

The synthesis of point clouds of multiple views increases the density of point cloud data, which is important to form LiDAR-like data. The 

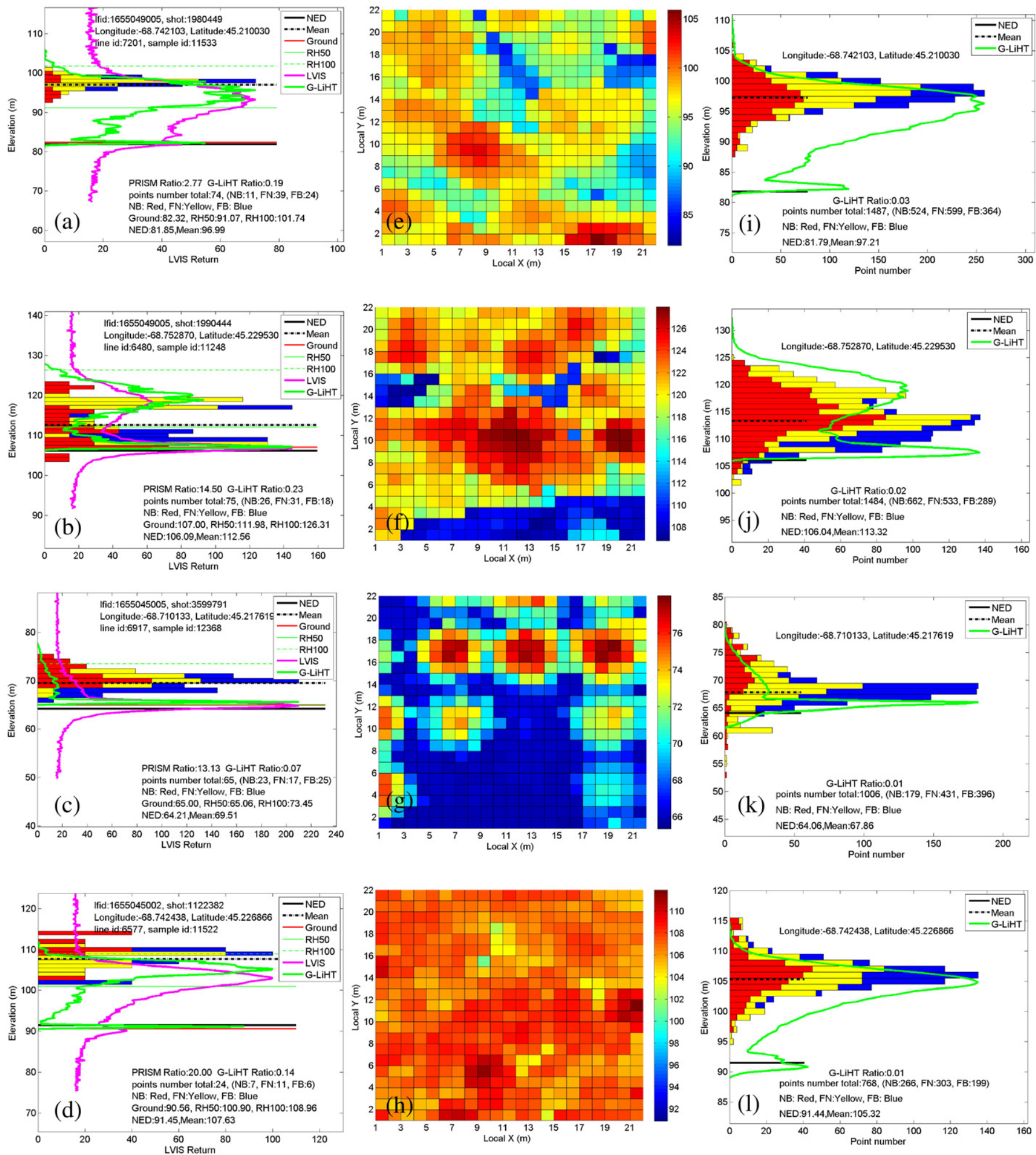

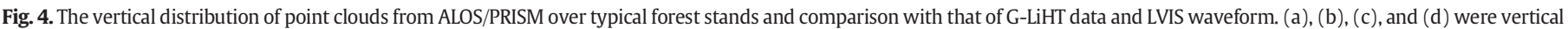

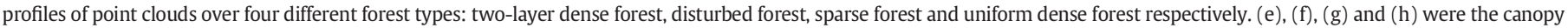

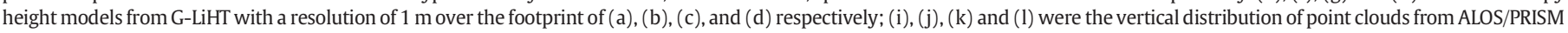

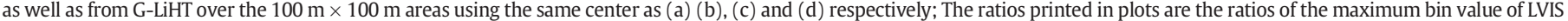
returns to PRISM or G-LiHT. The "ground", "RH50" and "RH100" were from LVIS data. The "Mean" was the average of all points of ALOS/PRISM within a pixel (21 m or 100 m).

effects are more obvious for sparse and unclosed forests. This is especially important for the mapping of forest structures using stereo imagery with relatively low spatial resolution such as PRISM data $(2.5 \mathrm{~m})$.
Some abnormal points from ALOS/PRISM point cloud data were observed over uniform forest areas. The influence of these points on the mean elevation was not severe but special care should be taken when 

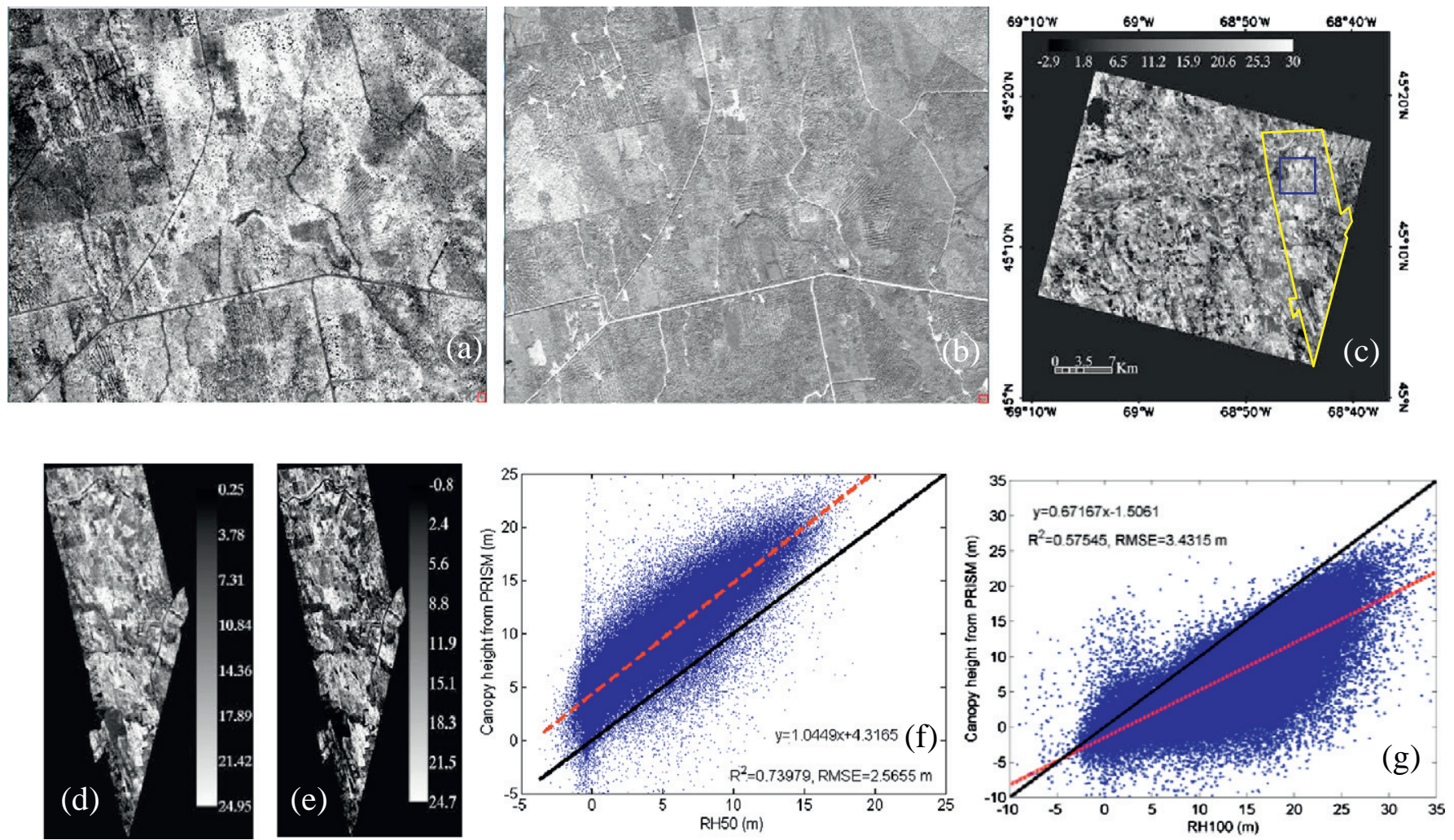

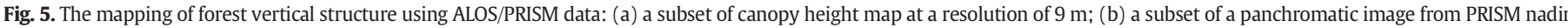

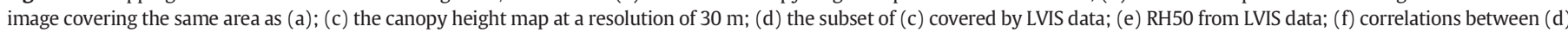
and (e); (g) correlations between (d) and RH100.

other height indices need to be derived from the vertical profiles of point clouds. The image texture may provide some information for the identification of these special cases.

A forest canopy height map was produced in this study from the synthesized point cloud data of ALOS/PRISM and NED. Fig. 5 exhibited the potential for the mapping of forest biomass using ALOS/PRISM and NED data. Studies by (Drake et al., 2002; Huang et al., 2013) have found that the RH50 has good linear correlation with biomass and it has been widely used for forest biomass estimation. Because less points near and at-ground surface were captured by ALOS/PRISM than LVIS, the PRISM height is systematically higher than RH50 (a bias of $4.3 \mathrm{~m}$ in Fig. 5(f)). The high correlation between the average height of PRISM point cloud and the RH50 indicates that the height data from synthesized point clouds of ALOS/PRISM could be used for forest biomass estimation.

\section{Conclusion}

Significant progress in digital photogrammetry makes the data processing easier than ever before. The features of point clouds from stereo imagery over forested area were investigated in this study taking the point clouds from small footprint LiDAR data and LiDAR waveforms as references. A method for the synergy of point clouds from multi-views was proposed and validated. Then the features of synthesized point clouds were examined. The results showed that the point clouds from different view combinations were complementary to each other spatially. A forest canopy height map was produced by subtracting NED from the averaged elevation of synthesized point clouds from stereo imagery. The canopy height map exhibited specific features of roads, forest edges and patches. The spatial pattern of canopy height map resembles that of LVIS RH50 very well. The linear regression showed that the canopy height map had a good correlation with RH50 of LVIS data with slope $=1.04$ and $\mathrm{R}^{2}=0.74$. This indicates that the combination of stereo imagery with NED has potential for mapping forest biomass.

The stereo imagery could see both canopy and ground surfaces in unclosed forest but only the canopy surface in the dense forest. Only the top layer could be captured for multi-layered dense forest. Due to obscured ground surface in the point clouds of stereo imagery, a positive bias was observed in comparison of PRISM height with LVIS RH50. The comparison with RH100 indicates that the PRISM height has some information on the top canopy height too. The point cloud from ALOS/PRISM exhibited interesting features in detecting forest vertical structures, which will be further explored in future studies.

\section{Acknowledgment}

This work was supported in part by the National Basic Research Program of China (Grant No. 2013CB733404), the National Natural Science Foundation of China (Grant Nos. 41001208, 40971203, 41171283, 91125003), National High-Tech R\&D Program of China (Grant. 2012AA12A306) and the Strategic Priority Research Program - Climate Change: Carbon Budget and Related Issues of the Chinese Academy of Sciences (Grant.XDA05050100). Portions of this work were supported by NASA's Terrestrial Ecology Program and the Hundred Talents Program of the Chinese Academy of Sciences.

\section{References}

Balzter, H., Rowland, C. S. \& Saich, P. (2007). Forest canopy height and carbon estimation at Monks Wood National Nature Reserve, UK, using dual-wavelength SAR interferometry. Remote Sensing of Environment, 108(3), 224-239.

Bortolot, Z. J. \& Wynne, R. H. (2005). Estimating forest biomass using small footprint LiDAR data: An individual tree-based approach that incorporates training data. ISPRS Journal of Photogrammetry and Remote Sensing, 59(6), 342-360.

Cloude, S. R., \& Papathanassiou, K. P. (2003). Three-stage inversion process for polarimetric SAR interferometry. IEE Proceedings - Radar Sonar and Navigation, 150(3), 125-134. 
Cook, B. D., Nelson, R. F., Middleton, E. M., Morton, D. C., McCorkel, J. T., Masek, J. G., et al. (2013). NASA Goddard's LiDAR, Hyperspectral and Thermal (G-LiHT) airborne imager. Remote Sensing, 5(8), 4045-4066.

Drake, J., Dubayah, R., Clark, D., Knox, R., Blair, J., Hofton, M., et al. (2002). Estimation of tropical forest structural characteristics using large-footprint LiDAR. Remote Sensing of Environment, 79(2-3), 305-319.

Dubayah, R. O., Sheldon, S. L., Clark, D. B., Hofton, M. A., Blair, J. B., Hurtt, G. C., et al. (2010). Estimation of tropical forest height and biomass dynamics using LiDAR remote sensing at La Selva, Costa Rica. Journal of Geophysical Research - Biogeosciences, 115.

Garestier, F., Dubois-Fernandez, P. C., \& Papathanassiou, K. P. (2008). Pine forest height inversion using single-pass X-band PolInSAR data. IEEE Transactions on Geoscience and Remote Sensing, 46(1), 59-68.

Gong, P., Mei, X. L., Biging, G. S., \& Zhang, Z. X. (2002). Improvement of an oak canopy model extracted from digital photogrammetry. Photogrammetric Engineering and Remote Sensing, 68(9), 919-924.

Hall, F. G., Bergen, K., Blair, J. B., Dubayah, R., Houghton, R., Hurtt, G., et al. (2011). Characterizing 3D vegetation structure from space: Mission requirements. Remote Sensing of Environment, 115(11), 2753-2775.

Hobi, M. L., \& Ginzler, C. (2012). Accuracy assessment of digital surface models based on WorldView-2 and ADS80 stereo remote sensing data. Sensors, 12(5), 6347-6368.

Huang, W. L., Sun, G. Q., Dubayah, R., Cook, B., Montesano, P., Ni, W. J., et al. (2013). Mapping biomass change after forest disturbance: Applying LiDAR footprint-derived models at key map scales. Remote Sensing of Environment, 134, 319-332.

Jan, A. (1993). Fundamentals of photointerpretation and photogrammetry. Islamabad: Pictorial Prints (Pvt) Ltd.

Kellndorfer, J., Walker, W., Pierce, L., Dobson, C., Fites, J. A., Hunsaker, C., et al. (2004). Vegetation height estimation from shuttle radar topography mission and national elevation datasets. Remote Sensing of Environment, 93(3), 339-358.

Korpela, I. (2004). Individual tree measurements by means of digital aerial Photogrammetry. Silva Fennica, 1-93.

Leberl, F., Irschara, A., Pock, T., Meixner, P., Gruber, M., Scholz, S., et al. (2010). Point clouds: LiDAR versus 3D vision. Photogrammetric Engineering and Remote Sensing, 76(10), 1123-1134.

Lefsky, M. A., Harding, D. J., Keller, M., Cohen, W. B., Carabajal, C. C., Espirito-Santo, F. D. et al. (2005). Estimates of forest canopy height and aboveground biomass using ICESat. Geophysical Research Letters, 32(22).

Naesset, E. (2002). Determination of mean tree height of forest stands by digital photogrammetry. Scandinavian Journal of Forest Research, 17(5), 446-459.

Neeff, T., Dutra, L. V., dos Santos, J. R., Freitas, C. D., \& Araujo, L. S. (2005). Tropical forest measurement by interferometric height modeling and P-band radar backscatter. Forest Science, 51(6), 585-594.

Ni, W., Sun, G., \& Ranson, K. J. (2013). Characterization of ASTER GDEM elevation data over vegetated area compared with LiDAR data. International Journal of Digital Earth, http://dx.doi.org/10.1080/17538947.17532013.17861025.

Ni, W., Sun, G., Zhang, Z., Guo, Z., \& He, Y. (2014a). Co-registration of two DEMs: Impacts on forest height estimation from SRTM and NED at mountainous areas. IEEE Geoscience and Remote Sensing Letters, 11(1), 273-277.
Ni, W., Sun, G., Zhang, Z., Guo, Z., \& He, Y. (2014b). A heuristic approach to reduce atmospheric effects in InSAR data for the derivation of digital terrain models or for the characterization of forest vertical structure. IEEE Geoscience and Remote Sensing Letters, 11(1), 268-272.

Ni-Meister, S. L. W., Strahler, A. H., Woodcock, C. E., Schaaf, C., Tian, Y., Ranson, J., et al. (2010). Assessing general relationships between above-ground biomass and vegetation structure parameters for improved carbon estimate from vegetation lidar. Journal of Geophysical Research, 115, G00E11.

Nuske, R. S., \& Nieschulze, J. (2004). The vegetation height as a tool for stand height determination: An application of automated digital photogrammetry in forestry. Allgemeine Forst Und Jagdzeitung, 175(1-2), 13-21.

Pang, Y., Lefsky, M., Miller, M., Sherrill, K., \& Andersen, H. (2008). Automatic tree crown delineation using discrete return LiDAR and its application in ICEsat vegetation product validation. Canadian Journal of Remote Sensing, 34(Suppl. 2), 471-484.

Papathanassiou, K. P. \& Cloude, S. R. (2001). Single-baseline polarimetric SAR interferometry. IEEE Transactions on Geoscience and Remote Sensing, 39(11), 2352-2363.

Sheng, Y. W., Gong, P., \& Biging, G. S. (2001). Model-based conifer-crown surface reconstruction from high-resolution aerial images. Photogrammetric Engineering and Remote Sensing, 67(8), 957-965.

Simard, M., Zhang, K. Q., Rivera-Monroy, V. H., Ross, M. S., Ruiz, P. L., Castaneda-Moya, E., et al. (2006). Mapping height and biomass of mangrove forests in Everglades National Park with SRTM elevation data. Photogrammetric Engineering and Remote Sensing, 72(3), 299-311.

Solberg, S., Astrup, R., Gobakken, T., Naesset, E., \& Weydahl, D. J. (2010). Estimating spruce and pine biomass with interferometric X-band SAR. Remote Sensing of Environment, 114(10), 2353-2360.

St-Onge, B., Jumelet, J., Cobello, M., \& Vega, C. (2004). Measuring individual tree height using a combination of stereophotogrammetry and LiDAR. Canadian Journal of Forest Research, 34(10), 2122-2130.

St-Onge, B., Vega, C., Fournier, R. A., \& Hu, Y. (2008). Mapping canopy height using a combination of digital stereo-photogrammetry and LiDAR. International Journal of Remote Sensing, 29(11), 3343-3364.

Sun, Y. H., Gong, H. L., Li, X. J., Pu, R. L., \& Li, S. A. (2011). Extracting eco-hydrological information of inland wetland from L-band Synthetic Aperture radar image in Honghe National Nature Reserve, Northeast China. Chinese Geographical Science, 21(2), 241-248.

Sun, G. Ranson, K. J. Kimes, D. S., Blair, J. B., \& Kovacs, K. (2008). Forest vertical structure from GLAS: An evaluation using LVIS and SRTM data. Remote Sensing of Environment, 112(1), 107-117.

Tao, C. V., \& Hu, Y. (2001). A comprehensive study of the rational function model for photogrammetric processing. Photogrammetric Engineering and Remote Sensing, 67(12), $1347-1357$

Toutin, T. (2004). DTM generation from Ikonos in-track stereo images using a 3D physical model. Photogrammetric Engineering and Remote Sensing, 70(6), 695-702. 